\title{
Lotteries, Sunspots, and Incentive Constraints ${ }^{1}$
}

\author{
Timothy J. Kehoe \\ Department of Economics, University of Minnesota, Minneapolis, Minnesota 55455 \\ Research Department, Federal Reserve Bank of Minneapolis, Minneapolis, Minnesota \\ 55480 \\ tkehoe (a) atlas.socsci.umn.edu \\ David K. Levine \\ Department of Economics, University of California Los Angeles, Los Angeles, CA 90095

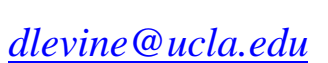 \\ and \\ Edward C. Prescott \\ Department of Economics, University of Minnesota, Minneapolis, Minnesota 55455 \\ Research Department, Federal Reserve Bank of Minneapolis, Minneapolis, Minnesota

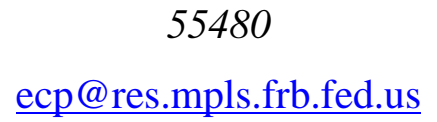

First version: September 11, 1997

This version: August 1, 2000

\begin{abstract}
We study a prototypical class of exchange economies with private information and indivisibilities. We establish an equivalence between lottery equilibria and sunspot equilibria and show that the welfare and existence theorems hold. To establish these results, we introduce the concept of the stand-in consumer economy, which is a standard convex, finite consumer, finite good, pure exchange economy. With decreasing absolute risk aversion and no indivisibilities, we prove that no lotteries are actually used in equilibrium. We provide a simple numerical example with increasing absolute risk aversion in which lotteries are necessarily used in equilibrium. We also show how the equilibrium allocation in this example can be implemented in a sunspot equilibrium.
\end{abstract}

\footnotetext{
${ }^{1}$ We are grateful to the participants at the Cowles Seminar at Yale University and at the Simposio de Análasis Económico. National Science Foundations Grants SBER 9618370, 9617899, and 9515256, and the UCLA Academic Senate provided financial support.
} 


\section{Introduction}

There is consideranle empirical evidence that, unlike in the standard complete markets model, individuals bear substantial idiosyncratic risk. See Kreuger [18] for a survey and discussion. Both incomplete markets models, such as those of Geanakoplos [13], and models of individual rationality constraints, such as those of Kehoe and Levine [15, 16], Kocherlakota [17], and Alvarez and Jermann [1], have been used to study idiosyncratic risk bearing. None of these models can explain a strong concentration of individual portfolios in a narrow range of assets. Why, for example, does Bill Gates hold largely Microsoft equity, or does a car dealer's portfolio consist largely of the dealership's inventory? While individuals with such undiversified portfolios are a small fraction of the population, they hold a large percentage of wealth.

Moral hazard is an obvious explanation for such undiversified portfolios, and recently there has been a resurgence of interest in introducing this feature into general equilibrium theory. Bennardo [3], Bennardo and Chiappori [4], and Bisin and Guaitoli [5] have been such efforts. The point of departure has been Prescott and Townsend [19, 20], who introduce both the idea that incentive constraints can be introduced into general equilibrium theory in a sensible way and the idea that lotteries play a potentially important role in the resulting theory. Although their theory has been widely used to study indivisibilities in the aggregate economy by Hansen [13], Rogerson [22], Cole and Prescott [9], and others, until recently little effort has been made to study incentive constraints from this point of view. ${ }^{2}$ The idea of using lotteries to study asset markets remains controversial.

This paper studies a prototypical class of incentive constrained environments in an effort to clarify a number of issues. One issue is how lottery equilibria are to be implemented. In the indivisibility case with a finite number of households, Shell and Wright [23] show that there is a close connection between lottery equilibria and sunspot equilibria, a connection that is made tight in Garratt et al. [12]. ${ }^{3}$ Here we show that

\footnotetext{
${ }^{2}$ A recent exception is Prescott and Townsend's [21] model of the firm.

${ }^{3}$ Shell and Wright [23] consider a model with complete information and a continuum of consumers. They show that every lottery equilibrium allocation can be decentralized as a sunspot equilibrium. In a model with complete information and a finite sunspot state space, Garratt [11] shows that, while every lottery equilibrium allocation can be decentralized as a sunspot equilibrium, not every sunspot equilibrium allocation is a lottery equilibrium allocation. Garratt et al. [12] establish the equivalence, in general
} 
similar results hold in the case of incentive constrained economies with a continuum of households. In the important case of decreasing absolute risk aversion and no indivisibilities, we show that lotteries are not actually needed in equilibrium. We provide a simple numerical example with increasing absolute risk aversion in which lotteries are necessarily used in equilibrium. We also show how the equilibrium allocation in this example can be implemented in a sunspot equilibrium. The proofs of theorems, the analysis of the example, and the computation of equilibria in these sorts of economies are greatly facilitated by the notion of the stand-in consumer economy. Overall, we argue that the Prescott and Townsend framework represents a sensible and useful framework for analyzing moral hazard and adverse selection in general equilibrium theory.

In recent related work, Cole and Kocherlakota [9] consider an environment like ours with private information over endowments. They show that, if storage is possible and unobservable by other housholds, then the equilibrium allocation is the same as that in an economy with an incomplete markets economy with a single asset that pays the same in all states.

\section{A Simple Insurance Problem}

There is a continuum [0,1] of households who are ex ante identical. There are two goods $j=1,2$. Let $c_{j}$ denote consumption of good $j$. Utility is given by $v_{1}\left(c_{1}\right)+v_{2}\left(c_{2}\right)$, where each $v_{i}(\cdot)$ is strictly concave and strictly increasing. The endowment of good 1 is risky, while good 2 has a certain endowment. Each household has an independent 50 percent chance of being in one of two states, $s \in\{g, b\}$. The endowment of good 1 is state dependent and can take on one of the two values $\omega_{1 g}$ and $\omega_{1 b}$, where $\omega_{1 g}>\omega_{1 b}$, while the endowment of good 2 is fixed at $\omega_{2}$.

Viewed in the aggregate, after the state is realized, half of the population has the good endowment, and half the bad endowment. After the state is realized, there are gains from trade, as the bad endowment households want to purchase good 1 and sell good 2. Before the state is realized, there are additional gains from trade since households want to purchase insurance against the bad state. In fact, since all households are ex ante identical and utility is strictly concave, the best symmetric allocation is that in which households 
consume $\left(\omega_{1 g}+\omega_{1 b}\right) / 2$ of good 1, and $\omega_{2}$ of good 2. Following the mechanism design literature, we refer to this allocation as the first best.

Suppose that the realization of the idiosyncratic risk is private information known only to the individual household. In this case, the first best allocation is not incentive compatible. In the first best allocation, bad endowment households receive an insurance payment of $\left(\omega_{1 g}-\omega_{1 b}\right) / 2$, while good endowment households must make a payment of the same amount. Consequently, good endowment households would misrepresent their endowment in order to receive a payment rather than make one.

One approach to modeling equilibrium is to prohibit trading in insurance contracts, and consider only trading that takes place ex post after the state is realized. This is an example of an incomplete markets model. The resulting competitive equilibrium leads to an equalization of marginal rates of substitution between the two goods for the two types of households, but there are unrealized gains from ex ante trade in insurance contracts.

A second approach to modeling equilibrium is to observe that it is possible to trade in insurance contracts provided that no household buys a contract that would later lead it to misrepresent its state. If endowments can be made public, but only voluntarily, then the good endowment household can imitate the bad endowment type, but not conversely. Suppose that a household attempts to purchase $\left(x_{1 b}, x_{2 b}\right)$ in state $\mathrm{b}$ in exchange for $\left(x_{1 g}, x_{2 g}\right)$ in state g. In the good endowment state, utility will be $v_{1}\left(\omega_{1 g}+x_{1 g}\right)+v_{2}\left(\omega_{2}+x_{2 g}\right)$. In this case, the good endowment household may want to pretend that the state is actually the bad endowment state, state $b$. To avoid detection, it must make the same spot market purchases that a bad endowment household would make, $\left(x_{1 b}, x_{2 b}\right)$. This results in utility $v_{1}\left(\omega_{1 g}+x_{1 b}\right)+v_{2}\left(\omega_{2}+x_{2 b}\right)$. Therefore the incentive compatibility constraint is

$$
v_{1}\left(\omega_{1 g}+x_{1 g}\right)+v_{2}\left(\omega_{2}+x_{2 g}\right) \geq v_{1}\left(\omega_{1 g}+x_{1 b}\right)+v_{2}\left(\omega_{2}+x_{2 b}\right) .
$$

If this constraint is satisfied, the good endowment household has no incentive to lie about its private information.

We now establish that, if trading in insurance contracts is prohibited, there are incentive compatible gains to allowing this trade. Let $\left(\hat{x}_{1 g}, \hat{x}_{2 g}, \hat{x}_{1 b}, \hat{x}_{2 b}\right)$ denote net trades by a household in an equilibrium in which trading in insurance contracts is prohibited. 
Since a bad endowment household cannot imitate a good endowment household, it faces no incentive constraints. Since the good endowment household could have purchased the net trade of the bad endowment household and had income left over, it strictly prefers its own net trade to that of the bad endowment household,

$$
v_{1}\left(\omega_{1 g}+\hat{x}_{1 g}\right)+v_{2}\left(\omega_{2}+\hat{x}_{2 g}\right)>v_{1}\left(\omega_{1 g}+\hat{x}_{1 b}\right)+v_{2}\left(\omega_{2}+\hat{x}_{2 b}\right) .
$$

We already know that $\left(\tilde{x}_{1 g}, \tilde{x}_{2 g}, \tilde{x}_{1 b}, \tilde{x}_{2 b}\right)=\left(-\left(\omega_{1 g}-\omega_{1 b}\right) / 2,0,\left(\omega_{1 g}-\omega_{1 b}\right) / 2,0\right)$ would be the equilibrium trades of insurance if there were no incentive constraints and would yield strictly higher ex ante utility than $\left(\hat{x}_{1 g}, \hat{x}_{2 g}, \hat{x}_{1 b}, \hat{x}_{2 b}\right)$ because utility is strictly concave. Consider the net trade

$$
\left(x_{1 g}, x_{2 g}, x_{1 b}, x_{2 b}\right)=\left((1-\theta) \hat{x}_{1 g}+\theta \tilde{x}_{1 g},(1-\theta) \hat{x}_{2 g}+\theta \widetilde{x}_{2 g},(1-\theta) \hat{x}_{1 b}+\theta \widetilde{x}_{1 b},(1-\theta) \hat{x}_{2 b}+\theta \widetilde{x}_{2 b}\right) .
$$

If $\theta$ is small enough, then good endowment household still has no incentive to misrepresent, but ex ante utility is strictly higher. Therefore, there are additional incentive compatible gains to trade that are not realized when trading in insurance contracts is prohibited.

Suppose, more generally, that households trade goods contingent on announcements. No household will ever deliver a bundle that is not incentive compatible. Every household knows this fact, and so only incentive compatible bundles can be traded. Notice, however, that this stronger argument does not guarantee that all incentive compatible bundles actually can be traded unless these contracts prohibit ex post trade: If a good endowment household can receive an insurance payment by claiming a bad endowment and then turn around and trade the insurance payment of good 1 for additional units of good 2, it will prefer this to admitting a good endowment. The contract must specifically prohibit households claiming to have a bad endowment from trading good 1 for good 2.

Contracts that preclude other trade are often referred to as exclusive contracts. Contracts of this type are common in insurance markets. Often insurance contracts specify that the insurance payment can be used only for a specific purpose, such as replacing a structure on a specific location. We consider only exclusive contracts in this paper.

Let $X$ denote the space of all net trades that satisfy the incentive constraint. Our program is to restrict households to trading plans in $X$ and then do ordinary competitive 
equilibrium theory. There are two complications with this program. First, fixing $\left(x_{1 g}, x_{2 g}\right)$, the set of $\left(x_{1 b}, x_{2 b}\right)$ that satisfies the incentive constraint fails to be convex, so $X$ is not a convex set. This means that Pareto improvements may be possible by using lotteries. Second, we can use lotteries to weaken the incentive constraints; that is, contingent on its announcement $s$, the household receives net trades $\left(x_{1 s}, x_{2 s}\right)$ that are random. Consequently, the incentive constraint need only hold in expected value. If we let $E_{i}$ denote the expectation conditional on the announcement of state $i$ the incentive constraint becomes

$$
E_{g}\left\{v_{1}\left(\omega_{1 g}+x_{1 g}\right)+v_{2}\left(\omega_{2 g}+x_{2 g}\right)\right\} \geq E_{b}\left\{v_{1}\left(\omega_{1 g}+x_{1 b}\right)+v_{2}\left(\omega_{2}+x_{2 b}\right)\right\} .
$$

For these two reasons, once we introduce incentive constraints into general equilibrium, we also introduce lotteries. ${ }^{4}$

\section{The Environment}

Households are of $I$ types $i=1, \ldots, I$. There is a continuum of ex ante identical households of each type. An individual household is denoted by $h \in H^{i}=\left[0, \lambda^{i}\right]$, where $\lambda^{i}>0$ is the size of the population of type $i$ households. A household's type is commonly known. There are $J$ traded goods $j=1, \ldots, J$. There are also two sources of uncertainty: a commonly observed sunspot and household specific idiosyncratic risk. A "sunspot" is a random variable $\sigma$ uniformly distributed on $[0,1]$. Idiosyncratic risk is represented by specifying that each household of type $i$ consumes in one of a finite number of states $s \in S^{i}$. Each state has probability $\pi_{s}^{i}>0$ where $\sum_{s \in S^{i}} \pi_{s}^{i}=1$. This probability has two interpretations: First, from the perspective of the individual household, it is the probability of being in the state $s$. Second, as we explain below, from the perspective of the entire population of households of type $i$, it represents the fraction of households in state $s$.

We assume that households can contract for delivery of goods contingent on the sunspot and the individual state of the household. ${ }^{5}$ We write $x_{j s}^{i}(h, \sigma) \in \Re$ for the net

\footnotetext{
${ }^{4}$ In addition to Prescott and Townsend [1984a, 1984b], such other authors as Arnott and Stiglitz [2] have remarked on the potential of lotteries to improve welfare.

${ }^{5}$ Strictly speaking, we should allow households to base contracts on the idiosyncratic states of other households. In the type of equilibrium we will consider contracts based on other household's idiosyncratic states do not serve any purpose. We omit them to avoid notational complication. This point is discussed further in Section 4.
} 
amount of good $j$ delivered to household $h$ of type $i$ when the idiosyncratic state is $s$ and the sunspot state is $\sigma$. The distribution of idiosyncratic shocks and sunspots are assumed to be independent. The idiosyncratic shocks are such that the aggregate net trade of all type $i$ households of good $j$ when the sunspot is $\sigma$ is given by

$$
y_{j}^{i}(\sigma)=\sum_{s \in S^{i}} \int \pi_{s}^{i} x_{j s}^{i}(h, \sigma) d h
$$

which is the assumption that $\pi_{s}^{i}$ is the fraction of households of type $i$ in state $s$. There are several justifications for this assumption. The easiest assumption is that idiosyncratic shocks are independent across households. It is known that this is inconsistent with aggregate net trades defined by Lesbesgue integration and a space of consumers on the unit interval; this is discussed in Boylan [6]. Alternatively, we could define aggregate net trades by the Pettis integral, as in Uhlig [24]. Or we could simply allow idiosyncratic states to be correlated across individuals. We prefer to avoid these technical issues, however, and simply justify the definition of aggregate consumption given as the limit of aggregate net trades in finite household economies with shocks independent across households. This, after all, is the purpose in introducing continuum economies in the first place.

Trading takes place before any uncertainty is realized. Then the idiosyncratic states are realized and announcements of states are made. Next, sunspots are realized. Finally, deliveries are made, no further trade is allowed, and consumption takes place. Notice that at this point we do not allow ex ante sunspots that are realized prior to the realization and announcement of individual states. A more general model would allow both ex ante and ex post sunspots. Later, we show that equilibria in the more general model are equivalent to equilibria in the ex post model we consider here. ${ }^{6}$

Fix household $h$ of type $i$. For each announcement of the idiosyncratic state $s$ and the sunspot $\sigma$ this household realizes a net trade $x_{s}^{i}(h, \sigma) \in \mathfrak{R}^{J}$. We assume that this net trade must belong to the feasible net trade set $X_{s}^{i}$. Notice that endowments are incorporated directly into the feasible net trade set and are not specified separately; we allow $X^{i}$ to depend on $s$ so that endowments may be idiosyncratic state contingent. In a

\footnotetext{
${ }^{6}$ In a model with only ex ante sunspots, equilibrium can be changed by adding ex post sunspots, because lotteries conditional on private information may be used to separate households with different risk preferences. This was originally pointed out by Cole [7].
} 
standard endowment economy, with endowments $\omega_{s}^{i}$, we have $X_{s}^{i}=\left\{x_{s}^{i} \mid x_{s}^{i} \geq-\omega_{s}^{i}\right\}$. Utility is given by $u_{s}^{i}: X_{s}^{i} \rightarrow \Re$. We use the notation

$$
x^{i}(h, \sigma) \in \Re^{J S^{i}}
$$

for the set of net trade vectors corresponding to different idiosyncratic states and

$$
u^{i}\left(x^{i}(h, \sigma)\right)=\sum_{s \in S^{i}} \pi_{s}^{i} u_{s}^{i}\left(x_{s}^{i}(h, \sigma)\right)
$$

for expected utility.

We next consider incentive constraints. These are derived from requiring that households not want to misrepresent private information about their own state. Private information about states is specified by sets of feasible reports $F_{s}^{i} \subseteq S^{i}$. These sets represent the reports that a household can make about his state when his true state is $s$ without being contradicted by either public information or physical evidence. Consequently, a set of feasible reports must satisfy two assumptions:

Feasible Truthtelling: For all $s \in S^{i}, s \in F_{s}^{i}$.

Feasible Misrepresentation: If $s^{\prime} \in F_{s}^{i}$, then $X_{s^{\prime}}^{i} \subseteq X_{s}^{i}$.

The second assumption requires that it is not possible to report a net trade set that is infeasible with respect to the true net trade set. This assumption rules out more complicated possibilities, such as situations where the feasibility of trading plans can only be discovered ex post and punishment imposed for violating contracts. In such a case, feasible reports would depend on the particular contract offered.

The notion of feasible reports that satisfy these two assumptions leaves substantial flexibility in building model economies. We provide two examples of classes of economies that satisfy our assumptions:

Public Endowments: The endowments $\omega_{s}^{i}$ are publicly observed, but preferences $u_{s}^{i}$ are not. Then $F_{s}^{i}=\left\{s^{\prime} \mid \omega_{s^{\prime}}^{i}=\omega_{s}^{i}\right\}$.

Voluntary Public Endowments: A household with endowment $\omega_{s}^{i}$ may publicly display any portion of its endowment. Preferences $u_{s}^{i}$ are private. By requiring that an endowment be displayed in order to be reported, we obtain $F_{s}^{i}=\left\{s^{\prime} \mid \omega_{s^{\prime}}^{i} \leq \omega_{s}^{i}\right\}$. In other words, households claiming a particular endowment can be required to display the 
claimed endowment, preventing households with smaller endowments from misrepresenting that they have larger endowments.

Notice that we treat sets of feasible reports as data. This avoids the deeper and more difficult question of how sets of feasible reports are generated from underlying fundamentals. Notice in particular that we could use the sets $F_{s}^{i}=\left\{s^{\prime} \mid \omega_{s^{\prime}}^{i} \leq \omega_{s}^{i}\right\}$ even in the case of public endowments. It is apparent that this would lead to smaller set of feasible allocations than making full use of the public information and taking $F_{s}^{i}=\left\{s^{\prime} \mid \omega_{s^{\prime}}^{i}=\omega_{s}^{i}\right\}$. A caveat to the results reported in this paper is that efficiency is conditional on a particular set of feasible reports.

A sunspot contingent trading plan $x^{i}(h, \sigma)$ is called incentive compatible if for all $s^{\prime} \in F^{i}(s)$

$$
\int u_{s}^{i}\left(x_{s}^{i}(h, \sigma)\right) d \sigma-\int u_{s}^{i}\left(x_{s}^{i}(h, \sigma)\right) d \sigma \geq 0 .
$$

We do not assume that $X_{s}^{i}$ is convex or that $u_{s}^{i}$ is concave or non-decreasing. We do assume:

Closed and Bounded Trades: $X_{s}^{i}$ is closed and bounded below.

Voluntary Trade: $0 \in X_{s}^{i}$.

Cheaper Point: For every $i$ and some $s \in S^{i}$ and $x_{s}^{i}<0, x_{s}^{i} \in \operatorname{Convex} \operatorname{Hull}\left\{X_{s}^{i}\right\}$.

Continuity: $u_{s}^{i}$ is continuous.

Non-satiation: From some state $s$ and all $x_{s}^{i} \in X_{s}^{i}$ there exists $\tilde{x}_{s}^{i} \in X_{s}^{i}$ such that $u_{s}^{i}\left(\tilde{x}_{s}^{i}\right)>u_{s}^{i}\left(x_{s}^{i}\right)$.

Boundary: If $\left\|x_{s}^{i}\right\| \rightarrow \infty$, then $\lim u_{s}^{i}\left(x_{s}^{i}\right) /\left\|x_{s}^{i}\right\|=0$.

With the exception of the boundary condition, these assumptions are self-explanatory. The boundary condition requires marginal utility to asymptote to zero; it says that eventually utility increases slower than any linear function. ${ }^{7}$

\footnotetext{
${ }^{7}$ Notice that nothing in these assumptions rule out indivisibilities, nor is it necessary to, as the literature on lotteries with indivisibilities discussed in the introduction shows.
} 
An important fact about the non-satiation condition is that it implies non-satiation for incentive compatible net trades.

Lemma 3.1: If $x^{i} \in \times_{s \in S^{i}} X_{s}^{i}$ is incentive compatible, then there is an incentive compatible $\tilde{x}^{i} \in \times_{s \in S^{i}} X_{s}^{i}$ such that $\sum_{s \in S^{i}} \pi_{s}^{i} u_{s}^{i}\left(\tilde{x}_{s}^{i}\right)>\sum_{s \in S^{i}} \pi_{s}^{i} u_{s}^{i}\left(x_{s}^{i}\right)$.

Proof: By the non-satiation assumption there is a state $s$ and $\tilde{x}_{s}^{i}$ with $u_{s}^{i}\left(\tilde{x}_{s}^{i}\right)>u_{s}^{i}\left(x_{s}^{i}\right)$. In other states $s^{\prime}$, if $\tilde{x}_{s}^{i} \notin X_{s^{\prime}}^{i}$ or if $\tilde{x}_{s}^{i} \in X_{s^{\prime}}^{i}$ and $u_{s^{\prime}}^{i}\left(\tilde{x}_{s}^{i}\right) \leq u_{s^{\prime}}^{i}\left(x_{s}^{i}\right)$, take $\tilde{x}_{s^{\prime}}^{i}=x_{s^{\prime}}^{i}$; otherwise, take $\tilde{x}_{s^{i}}^{i}=\tilde{x}_{s}^{i}$. Clearly, $\sum_{s \in S^{i}} \pi_{s}^{i} u_{s}^{i}\left(\tilde{x}_{s}^{i}\right)>\sum_{s \in S^{i}} \pi_{s}^{i} u_{s}^{i}\left(x_{s}^{i}\right)$ because $\pi_{s}^{i}>0$.

We now argue that $\tilde{x}^{i}$ is incentive compatible. Suppose $s^{\prime \prime} \in F_{s^{\prime}}$. Since $u_{s^{\prime}}^{i}\left(x_{s^{\prime}}^{i}\right) \geq u_{s^{\prime}}^{i}\left(x_{s^{\prime \prime}}^{i}\right)$, it follows that $u_{s^{\prime}}^{i}\left(\tilde{x}_{s^{\prime}}^{i}\right) \geq u_{s^{\prime}}^{i},\left(\tilde{x}_{s^{\prime \prime}}^{i}\right)$ holds if $\tilde{x}_{s^{\prime \prime}}^{i}=x_{s^{\prime \prime}}^{i}$. If $\tilde{x}_{s^{\prime \prime}}^{i} \neq x_{s^{\prime \prime}}^{i}$ then $\tilde{x}_{s^{\prime \prime}}^{i}=\tilde{x}_{s}^{i}$. If $u_{s^{\prime}}^{i},\left(x_{s^{i}}^{i}\right) \geq u_{s^{i}}^{i}\left(\tilde{x}_{s^{\prime \prime}}^{i}\right)$, since $u_{s^{\prime}}^{i},\left(\tilde{x}_{s^{\prime}}^{i}\right) \geq u_{s^{i}}^{i}\left(x_{s^{\prime}}^{i}\right)$, the incentive constraint holds. If $u_{s^{\prime}}^{i}\left(x_{s^{\prime}}^{i}\right)<u_{s^{\prime}}^{i},\left(\tilde{x}_{s^{\prime \prime}}^{i}\right)$, we have $u_{s^{\prime}}^{i}\left(x_{s^{\prime}}^{i}\right)<u_{s^{\prime}}^{i}\left(\tilde{x}_{s^{\prime \prime}}^{i}\right)=u_{s^{\prime}}^{i}\left(\tilde{x}_{s}^{i}\right)$, implying that $\tilde{x}_{s^{\prime}}^{i}=\tilde{x}_{s}^{i}$. By our construction of $\tilde{x}_{s}^{i}$, again the incentive constraint holds.

Three points to emphasize about the model are

- Types are commonly known; the idiosyncratic states may or may not be private information. It is important that contracting takes place prior to learning any private information. If contracting is possible only after learning private information, or, what amounts to the same thing, if types are private information, then incentives to misrepresent information will depend on the net trades of rival households. This represents an externality that may invalidate the welfare theorems. ${ }^{8}$

- Households do not care about the private information of rival households. This assumption could be relaxed, but it would then be necessary to allow contracting based upon the announcements of the relevant rivals.

- We have implicitly assumed that contracts are exclusive - that is, that trading is not possible after deliveries are made. As we noted in the example, equilibrium with nonexclusive trading is quite different than with exclusivity. As pointed out by Prescott and Townsend [19], the welfare theorems can fail without exclusivity.

\footnotetext{
${ }^{8}$ Prescott and Townsend [1984b] give an example in which the first welfare theorem holds, but the second fails. In other examples, such as those in Prescott and Townsend [1984a], both welfare theorems fail and equilibrium may not exist.
} 
We conclude this section by illustrating how the example of the previous section fits into this framework. In the example $I=1, J=2, \lambda^{1}=1, S^{1}=\{g, b\}, \pi_{b}^{1}=\pi_{g}^{1}=1 / 2$. The net trade sets are those net trades that exceed the negative of the endowment

$$
X_{s}^{1}=\left\{\left(x_{1 s}^{1}, x_{2 s}^{1}\right) \mid x_{1 s}^{1} \geq-\omega_{1 s}, x_{2 s}^{1} \geq-\omega_{2}\right\} .
$$

The utility function for net trades is derived from the utility of consumption according to

$$
\begin{aligned}
& u_{s}^{1}\left(x_{1 s}^{1}, x_{2 s}^{1}\right)=v_{1}\left(\omega_{1 s}+x_{1 s}^{1}\right)+v_{2}\left(\omega_{2}+x_{2 s}^{1}\right) \\
& u^{1}\left(x_{1 g}^{1}, x_{2 g}^{1}, x_{1 b}^{1}, x_{2 b}^{1}\right)=\frac{1}{2} u_{g}^{1}\left(x_{1 g}^{1}, x_{2 g}^{1}\right)+\frac{1}{2} u_{b}^{1}\left(x_{1 b}^{1}, x_{2 b}^{1}\right) .
\end{aligned}
$$

The feasible reporting sets reflect the fact that $X_{b}^{1} \subset X_{g}^{1}$,

$$
F_{b}^{1}=\{b\}, \quad F_{g}^{1}=\{g, b\} .
$$

There is one incentive constraint, corresponding to a good endowment state reporting a bad endowment:

$$
\int u_{g}^{1}\left(x_{g}^{1}(h, \sigma)\right) d \sigma-\int u_{g}^{1}\left(x_{b}^{1}(h, \sigma)\right) d \sigma \geq 0 .
$$

\section{Equilibrium with Sunspots}

A sunspot allocation is a measurable map for each type from households to individual trading plans; that is $x_{s}^{i}(h, \sigma) \in X_{s}^{i}$. An allocation is socially feasible if for each sunspot realization $\sigma$

$$
\sum_{i=1}^{I} \lambda^{i} \sum_{s \in S^{i}} \int \pi_{s}^{i} x_{s}^{i}(h, \sigma) d h \leq 0 .
$$

Notice that this definition incorporates public free disposal; we do not assume individuals can secretly dispose of goods. We say that an allocation has equal utility if for each type $\int u^{i}\left(x^{i}(h, \sigma)\right) d \sigma=\int u^{i}\left(x^{i}\left(h^{\prime}, \sigma\right)\right) d \sigma$ for almost all $h, h^{\prime}$.

Let $e$ denote the idiosyncratic states of all households of all types; $e(i, h)$ is the idiosyncratic state of household $h$ of type $i$. Then the Arrow-Debreu commodity is a delivery of $j$ goods contingent on $(e, \sigma)$. Arrow-Debreu prices are $q_{(e, \sigma)} \in \mathfrak{R}_{+}^{J}$. Because there is no aggregate risk, we restrict attention to Arrow-Debreu equilibria in which prices are independent of $e$; that is, $q_{(e, \sigma)}=q(\sigma)$. With these prices, the cost to a household $h$ of type $i$ to purchase $x_{s}^{i}$ at $\sigma$ is

$$
\int_{\{e \mid e(i, h)=s\}} q_{(e, \sigma)} \cdot x_{s}^{i} d e=\int_{\{e \mid e(i, h)=s\}} q(\sigma) \cdot x_{s}^{i} d e=\pi_{s}^{i} q(\sigma) \cdot x_{s}^{i} .
$$


We refer to a non-zero measurable function $q(\sigma) \in \mathfrak{R}_{+}^{J}$ as a price function.

A sunspot equilibrium with transfers consists of a socially feasible sunspot allocation $\hat{x}$ together with a price function $\hat{q}$. For all types $i$ and almost all $h \in\left[0, \lambda^{i}\right]$, $\hat{x}^{i}(h$,$) must maximize \int u^{i}\left(x^{i}(h, \sigma)\right) d \sigma$ over sunspot contingent trading plans $x^{i}(h, \cdot)$ satisfying the sunspot budget constraint

$$
\int \sum_{s \in S^{i}} \pi_{s}^{i} \hat{q}(\sigma) \cdot x_{s}^{i}(h, \sigma) d \sigma \leq \int \sum_{s \in S^{i}} \pi_{s}^{i} \hat{q}(\sigma) \cdot \hat{x}_{s}^{i}(h, \sigma) d \sigma,
$$

and the incentive constraints $\int\left[u_{s}^{i}\left(x_{s}^{i}(h, \sigma)\right)-u_{s}^{i}\left(x_{s^{\prime}}^{i}(h, \sigma)\right)\right] d \sigma \geq 0, s^{\prime} \in F^{i}(s)$. The transfers themselves must satisfy the equal treatment condition that they depend only on types:

$\int \sum_{s \in S^{i}} \pi_{s}^{i} \hat{q}(\sigma) \cdot \hat{x}_{s}^{i}(h, \sigma) d \sigma=\int \sum_{s \in S^{i}} \pi_{s}^{i} \hat{q}(\sigma) \cdot \hat{x}_{s}^{i}\left(h^{\prime}, \sigma\right) d \sigma$ for almost all $h, h^{\prime}$.

A sunspot equilibrium is a sunspot equilibrium with transfers in which the transfers are zero:

$$
\int \sum_{s \in S^{i}} \pi_{s}^{i} \hat{q}(\sigma) \cdot \hat{x}_{s}^{i}(h, \sigma) d \sigma=0 \text { for almost all } h .
$$

Finally, a sunspot allocation is Pareto efficient if there is no alternative socially feasible allocation satisfying the incentive constraints in which almost all households have no less utility and a positive measure of households have strictly more utility.

An immediate consequence of the fact that the transfers satisfy the equal treatment condition is the conclusion that the equilibrium allocation must be an equal utility allocation. If it were not, then a positive measure of type $i$ could increase their utility by switching to a consumption plan used by others of the same type.

Lemma 4.1 A sunspot equilibrium allocation with transfers is an equal utility allocation.

Our main goal is to establish the main theorems of competitive general equilibrium theory for the sunspot economy

Theorem 4.2 (First Welfare Theorem) Every sunspot equilibrium allocation with transfers is Pareto efficient.

Theorem 4.3 (Second Welfare Theorem) For every Pareto efficient allocation $x$ with equal utility there are prices $q$ such that $(x, q)$ are a sunspot equilibrium with transfers.

Theorem 4.4 (Existence Theorem) There is at least one sunspot equilibrium. 
The first welfare theorem is a relatively direct consequence of the non-satiation assumption and the standard proof of the first welfare theorem. The remaining results follow from equivalence theorems below.

\section{Equilibrium with Lotteries}

A probability distribution $\mu_{s}^{i}$ over $X_{s}^{i}$ is referred to as a lottery. We define

$$
u_{s}^{i}\left(\mu_{s}^{i}\right)=\int u_{s}^{i}\left(x_{s}^{i}\right) d \mu_{s}^{i}\left(x_{s}^{i}\right)
$$

as the expected utility from the lottery. From the point of view of individual utility, all trading plans that induce the same set of lotteries

$$
\mu^{i}=\left\{\mu_{s}^{i}\right\}_{s \in S^{i}}
$$

yield the same utility, and the incentive constraints can also be computed directly from the lottery. The aggregate resources used by a set of lotteries are

$$
y^{i}=\lambda^{i} \sum_{s \in S^{i}} \pi_{s}^{i} \int x_{s}^{i} d \mu_{s}^{i}\left(x_{s}^{i}\right) .
$$

Notice that with a continuum of households we need not distinguish between realized and expected net trades. This distinction is important in decentralizing lotteries in the indivisible case with a finite number of households, as can be seen in the work of Garratt [11] and Garratt et al. [12].

To illustrate our notation we apply it our insurance example. In the example, there are two states $S^{1}=\{g, b\}$, and two net trading sets $X_{s}^{1}$ consisting of net trades that are at least as great as the negative of the endowment. The set of lotteries has one lottery corresponding to each state $\mu_{s}^{1}$. The lotteries $\mu_{s}^{1}$ are each non-negative measures that satisfy

$$
\int d \mu_{s}^{1}\left(x_{s}^{1}\right)=1
$$

We now consider the Prescott and Townsend perspective, in which households trade directly in lotteries. Our goal is to show that this formulation is equivalent to the sunspot formulation. 
A lottery allocation is a vector of sets of lotteries, $\mu=\left(\mu^{1}, \ldots, \mu^{I}\right)$, where the set of lotteries $\mu^{i}$ assigns lotteries $\mu_{s}^{i}$ to type $i$ in each idiosyncratic state. ${ }^{9}$ Notice that this requires all households of a given type to purchase the same lottery. Because preferences are convex over lotteries, it makes sense to impose this restriction and we demonstrate in the next section that there is no loss of generality in this. A set of lotteries is socially feasible if

$$
\sum_{i=1}^{I} \lambda^{i} \int \sum_{s \in S^{i}} \pi_{s}^{i} x_{s}^{i} d \mu_{s}^{i}\left(x^{i}\right) \leq 0
$$

This says that in the aggregate the expected net trades used by the lottery allocation is non-positive.

A lottery allocation is Pareto efficient if no socially feasible, incentive compatible Pareto improvement is possible. A lottery equilibrium with transfers consists of a socially feasible lottery allocation $\hat{\mu}$ together with non-zero price vector $\hat{p} \in \mathfrak{R}_{+}^{J}$. For all types $i$, $\hat{\mu}^{i}$ must maximize

$$
\sum_{s \in S^{i}} \pi_{s}^{i} u_{s}^{i}\left(\mu_{s}^{i}\right)
$$

over lotteries $\mu^{i}$ satisfying the lottery budget constraint

$$
\hat{p} \cdot \int \sum_{s \in S^{i}} \pi_{s}^{i} x_{s}^{i} d \mu_{s}^{i}\left(x_{s}^{i}\right) \leq \hat{p} \cdot \int \sum_{s \in S^{i}} \pi_{s}^{i} x_{s}^{i} d \hat{\mu}_{s}^{i}\left(x_{s}^{i}\right),
$$

and the incentive constraints $u_{s}^{i}\left(\mu_{s}^{i}\right)-u_{s}^{i}\left(\mu_{s^{\prime}}^{i}\right) \geq 0, s^{\prime} \in F^{i}(s)$. A lottery equilibrium is a lottery equilibrium with transfers in which the transfers are zero

$$
\hat{p} \cdot \int \sum_{s \in S^{i}} \pi_{s}^{i} x_{s}^{i} d \mu_{s}^{i}\left(x_{s}^{i}\right)=0 .
$$

Notice that in this formulation, lotteries are priced according to the aggregate resources they use. This is a no-arbitrage condition: two lotteries that use the same aggregate resources must have the same price. If one lottery uses aggregate resources $y$ and another $\hat{y}$, and if the cost of buying $y$ and $\hat{y}$ separately exceeds the cost of buying $y+\hat{y}$, it would be profitable to buy the joint lottery $y+\hat{y}$ and sell the pieces, while in the opposite case, the pieces should be bought separately, then packaged and sold. Only

\footnotetext{
${ }^{9}$ We could have equally well followed the formalism of defining a trade vector $\mu=\left(\mu^{1}, \ldots, \mu^{I}\right)$, and restricting households of type $i$ to the trade sets in which the lottery vectors for all other types put probability 0 on all trades. The notation followed here has the advantage of being less cumbersome, but has the disadvantage of implicitly having different trade spaces, and underlying commodity spaces, for different household types.
} 
linear pricing in the aggregate resources guarantees that there are no arbitrage opportunities. $^{10}$

Let $k^{i}=\sum_{s \in S^{i}}\left(\# F_{s}^{i}-1\right)$ denote the number of incentive constraints. There are four basic features of lotteries that are worth emphasizing:

\section{Lemma 5.1}

(a) A convex combination of incentive compatible sets of lotteries is incentive compatible.

(b) Let $y^{i}, \tilde{y}^{i}$ be the resources used by the sets of lotteries $\mu^{i}, \tilde{\mu}^{i}$ and let $0 \leq \alpha \leq 1$. Then the set of lotteries $\alpha \mu^{i}+(1-\alpha) \tilde{\mu}^{i}$ uses aggregate resources $\alpha y^{i}+(1-\alpha) \tilde{y}^{i}$.

(c) For any incentive compatible set of lotteries $\mu^{i}$ there is another incentive compatible set of lotteries $\tilde{\mu}^{i}$ using the same aggregate resources, yielding the same utility, and each lottery $\tilde{\mu}_{s}^{i}$ having support on $k^{i}+J+2$ points.

(d) Let $y^{i}$ be the resources used by the incentive compatible set of lotteries $\mu^{i}$, and suppose $\tilde{y}^{i}>y^{i}$. Then there is an incentive compatible set of lotteries $\tilde{\mu}^{i}$ using no more resources than $\tilde{y}^{i}$ that yields strictly more utility than $\mu^{i}$.

Proof: (a) and (b) are immediate. The proof of (c) is largely mathematical, and is provided in the Appendix. To prove (d), first apply (c) to find an incentive compatible lottery $\tilde{\widetilde{\mu}}^{i}$ with finite support yielding the same utility as $\mu^{i}$. Because this lottery has finite support, it follows from Lemma 3.1 that there is an incentive compatible net trade $x^{i} \quad$ with $\sum_{s \in S^{i}} \pi_{s}^{i} u_{s}^{i}\left(x_{s}^{i}\right)>\sum_{s \in S^{i}} \pi_{s}^{i} u_{s}^{i}\left(\tilde{\widetilde{\mu}}_{s}^{i}\right)=\sum_{s \in S^{i}} \pi_{s}^{i} u_{s}^{i}\left(\mu_{s}^{i}\right)$. Let $\tilde{\mu}_{s}^{i}$ be the degenerate lottery with point mass on $x_{s}^{i}$. Then for all $0<\alpha \leq 1$, the set of lotteries $(1-\alpha) \mu^{i}+\alpha \tilde{\mu}^{i}$ is incentive compatible by (a) and yields strictly more utility than $\mu^{i}$. As $\alpha$ approaches 0 , however, the aggregate resources used by this set of lotteries approach $y^{i}$ and, therefore, for $\alpha$ sufficiently small, are less than $\tilde{y}^{i}$.

\footnotetext{
${ }^{10}$ Much of the literature on lotteries studies production economies in which firms can repackage lotteries into different lotteries using the same resources; what we refer to as a no-arbitrage condition follows in that setting from profit maximization by firms. Our approach follows Hansen [1985].
} 
We will establish the main theorems of competitive general equilibrium theory for the lottery economy, as well as the sunspot economy.

Theorem 5.2 (First Welfare Theorem) Every lottery equilibrium allocation with transfers is Pareto efficient.

Theorem 5.3 (Second Welfare Theorem) For every Pareto efficient allocation there are prices forming a lottery equilibrium with transfers.

Theorem 5.4 (Existence Theorem) There is at least one lottery equilibrium.

In Prescott and Townsend [20], these theorems are proved directly; we give alternative proofs below. Our results on sunspot equilibria then follow from showing that lottery and sunspot allocations are equivalent.

\section{Sunspot Equilibrium versus Lottery Equilibrium}

Sunspot allocations and lottery allocations are different descriptions of randomization. For example, suppose that there are two identical types, and one good, automobiles, for which the consumption vector is either one automobile or zero. Suppose moreover, that each type is endowed with one half an automobile per capita. From the lottery perspective, the situation is simple: there can be no trade between the two types, so each household should receive an automobile with probability $1 / 2$. In other words, in equilibrium, each household of each type purchases a lottery with a 50 percent chance of 1 automobile, and a 50 percent chance of 0 automobiles. In our notation, $\mu^{i}(1 / 2)=1 / 2$ and $\mu^{i}(-1 / 2)=1 / 2$ where $\omega^{i}=1 / 2$ and $\omega^{i}+x^{i}$ is equal to 1 or 0 for both household types $i=1,2$.

This lottery can be implemented in many ways by means of sunspots. For example, we could imagine that the individual lotteries are independent, ${ }^{11}$ and that in the aggregate the strong law of large number leads to social feasibility. An alternative formulation would be to have a simple sunspot allocation in which when the sunspot variable satisfies $\sigma \leq 1 / 2$, the first type receives all the cars and, when $\sigma>1 / 2$, the second type receives all the cars. From an individual point of view it makes no difference which of these methods is used to allocate cars.

\footnotetext{
${ }^{11}$ Subject to usual caveat about a continuum of independent random variables; see the discussion above.
} 
A sunspot allocation may induce different lotteries for different households. To get a single set of lotteries for each type, as required for a lottery allocation, we average together the household specific lotteries. Begin with a sunspot allocation $\tilde{x}(h, \sigma)$. For each household, there correspond lotteries $\mu_{s}^{i}\left(\tilde{x}_{s}^{i}(h, \cdot)\right)$. We can then average these lotteries over households to get a mean lottery for the entire type $\tilde{\mu}_{s}^{i}=\int \mu_{s}^{i}\left(\tilde{x}_{s}^{i}(h, \cdot)\right) d h / \lambda^{i}$. Notice that the resources used by this lottery are equal to the expected resources used by the sunspot allocation; that is,

$$
\int x_{s}^{i} d \tilde{\mu}_{s}^{i}\left(x_{s}^{i}\right)=\int \tilde{x}_{s}^{i}(h, \sigma) d h d \sigma / \lambda^{i} .
$$

Moreover, by definition, in an equal utility sunspot allocation households of type $i$ must be indifferent between the allocations $\tilde{x}^{i}(h, \cdot)$ and $\tilde{x}^{i}\left(h^{\prime}, \cdot\right)$ for almost all $h, h^{\prime}$. Since their utility is linear in probabilities, this means they must be indifferent between $\tilde{x}^{i}(h, \cdot)$ and the mean set of lotteries $\tilde{\mu}^{i}$ for almost all $h$. In a similar vein, since the incentive constraints hold for almost all individual sets of lotteries and are also linear in probabilities, the mean set of lotteries must satisfy the incentive constraint. Consequently, the mean set of lotteries corresponding to a sunspot equilibrium allocation is a natural candidate to be an equilibrium of the lottery economy. We provide an example of averaging of lotteries in Section 10.

If $q(\sigma)$ is a price function in the sunspot economy, we can in a similar way define the mean price $\bar{q}=\int q(\sigma) d \sigma$. Although it is not obvious, we will show below that the mean price is in fact a correct way to price the mean lottery in the lottery economy. $^{12}$

To appreciate the possibility of $q(\sigma)$ not being constant, consider a variant of our automobile example. Again there are two identical types, but now there are two goods, clothes washers and dryers. Households of each type are endowed with 1/2 unit each of washers and dryers and can consume only 1 or 0 of each of these goods. Furthermore, these goods are optimally be consumed in fixed proportions:

$$
u^{i}\left(x_{1}^{i}, x_{2}^{i}\right)=\min \left[\omega_{1}^{i}+x_{1}^{i}, \omega_{2}^{i}+x_{2}^{i}\right] .
$$

\footnotetext{
${ }^{12}$ Alternatively, we could simply require that in a sunspot allocation each household of a type have the same lottery. While this restriction seems natural in the context of the lottery model with a representative household, it does seem as natural in the sunspot model. Fortunately, we can show that only the mean lottery and mean price matter.
} 
It is easy to check that one equilibrium is where $q(\sigma)=q=(1,0)$ and

$$
x^{1}(h, \sigma)= \begin{cases}(1 / 2,1 / 2) & \text { if } \sigma \leq 1 / 2 \\ (-1 / 2,-1 / 2) & \text { if } \sigma>1 / 2,\end{cases}
$$

with the opposite allocation to households of type 2. Another equilibrium has the same allocation but the price vector $q(\sigma)=q=(0,1)$. Yet another equilibrium would have

$$
q(\sigma)= \begin{cases}(1,0) & \text { if } \sigma \leq 1 / 4 \text { or } \sigma>3 / 4 \\ (0,1) & \text { if } 1 / 4<\sigma \leq 3 / 4\end{cases}
$$

The possibility of prices $q(\sigma)$ that vary with the sunspot $\sigma$ arises because there is more that one constant price vector that can support an allocation. Notice, in our example, that the mean price $\bar{q}=(1 / 2,1 / 2)$ is also an equilibrium price. In Theorem 6.2 , we prove that this is true in general. In our example, in which the role for sunspots arises because of indivisibilities, the possibility of more than one constant price vector supporting an allocation is degenerate in that it disappears if we perturb the endowments. In economies where the role for sunspots arises because of incentive constraints, however, there is no need for equilibria with more than one supporting price to be degenerate.

We define a sunspot allocation to be equivalent to a lottery allocation if for each type the mean set of lotteries of the sunspot allocation is equal to the corresponding set of lotteries in the lottery allocation. We define sunspot prices to be equivalent to a lottery price if the mean price of the sunspot prices is equal to the lottery price. By definition, there is only one lottery allocation and price that is equivalent to a given sunspot allocation and price function. As we have already noted, however, there is not a unique way to construct a sunspot allocation (or prices) from a lottery allocation. Nevertheless, there is one important construction that plays a key theoretical role in moving from lottery economies to sunspot economies. For a given lottery price $p$ we define the constant function $q(\sigma)=p$ to be the canonical sunspot price function ${ }^{13}$. For a given lottery allocation $\mu$ we define the canonical sunspot allocation to be a particular allocation in which the aggregate resources used by each type are independent of the sunspot state. Specifically, corresponding to the lottery $\mu_{s}^{i}$ is a random variable $\tilde{x}_{s}^{i}(\sigma)$. Recall that

\footnotetext{
${ }^{13}$ Garratt et al. [12] call these prices constant probability adjusted prices. They show that in economies with complete information all sunspot equilibrium allocations can be supported by prices that are collinear with probabilities if the sunspot randomization device is continuous.
} 
$a$ mod $b$ is the remainder of $a$ divided by $b$. We define the canonical sunspot allocation as $x_{s}^{i}(h, \sigma)=\tilde{x}_{s}^{i}\left((\sigma+h) \bmod \lambda^{i}\right) .{ }^{14}$ Notice that at this canonical allocation, the aggregate net trades by all households of a type is independent of the realization of the sunspot.

These simple constructions show that for every lottery allocation and price there is at least one equivalent sunspot allocation and price. Because the construction of the lottery allocation preserves utility, social feasibility and the incentive constraints, we can draw an immediate conclusion about Pareto efficiency.

Theorem 6.1 An equal utility allocation is Pareto efficient in the sunspot economy if and only if any (or all) equivalent allocations in the lottery economy are Pareto efficient. Moreover, the socially feasible, incentive compatible equal utility set in the sunspot economy is the same as the socially feasible incentive compatible utility set in the lottery economy.

Less immediately obvious is the equivalence of equilibria in the two economies.

Theorem 6.2 An allocation and price are an equilibrium with transfers in the lottery economy if any (or all) equivalent allocation and price functions are an equilibrium with transfers in the sunspot economy. An allocation and constant price function are an equilibrium with transfers in the sunspot economy if the equivalent allocation and price function are an equilibrium with transfers in the lottery economy. In both cases the size of the transfers is the same in the two economies.

Proof: Consider a sunspot allocation $\hat{x}$ and price function $\hat{q}$ and an equivalent lottery allocation $\hat{\mu}$ and price $\hat{p}$. Suppose first that $\hat{q}$ is constant (in particular, that $\hat{q}(\sigma)=\hat{p}$ ) and that $\hat{\mu}, \hat{p}$ are an equilibrium with transfers in the lottery economy. Since households care only about their individual lottery and since $\hat{q}(\sigma)=\hat{p}, \hat{x}, \hat{q}$ are an equilibrium with transfers in the sunspot economy. Since in both cases each type pays only for the aggregate resources used, which is the same in both economies, the transfers must be the same in both cases.

Now suppose instead that $\hat{x}, \hat{q}$ are an equilibrium with transfers in the sunspot economy, and that possibly $\hat{q}$ is not constant. We must show that $\hat{\mu}, \hat{p}$ are a lottery

\footnotetext{
${ }^{14}$ There are many ways of mapping a lottery allocation into a sunspot allocation involving different ways of correlating outcomes across individuals. See Shell and Wright [23] and Garratt et al. [12] for discussions.
} 
equilibrium with transfers. To show this we must show that for each type $i$ any set of lotteries $\mu^{i}$ that yield more utility than $\hat{\mu}^{i}$ cannot be afforded at the prices $\hat{p}$ and that $\hat{\mu}^{i}$ can be purchased at those prices.

First, we show that for each type $i$, any set of lotteries $\mu^{i}$ that yield more utility than $\hat{\mu}^{i}$ cannot be afforded at the prices $\hat{p}$. Suppose that $\mu^{i}$ is in fact affordable and yields more utility than $\hat{\mu}^{i}$. Notice that since they are equivalent, the utility from $\hat{\mu}^{i}$ is the same utility $\hat{x}^{i}$ gives almost all households. We use $\mu^{i}$ to construct a sunspot plan that is affordable at prices $\hat{q}$ yielding the same utility as $\hat{\mu}^{i}$; this will be the desired contradiction. Consider the canonical sunspot allocation $x^{i}$ corresponding to $\mu^{i}$. This gives every household in $i$ more utility than $\hat{x}^{i}$. It is also constructed so that $\int x_{s}^{i}(h, \sigma) d h$ is independent of $\sigma$. By construction it is affordable at prices $q(\sigma)=\hat{p}$; because $\hat{p}$ is

the average of $\hat{q}(\sigma)$ and $\int x_{s}^{i}(h, \sigma) d h$ is independent of $\sigma$, it is therefore affordable at prices $\hat{q}(\sigma)$. It follows that for a positive measure set of household $h, x^{i}(h, \cdot)$ is also affordable at prices $\hat{q}(\sigma)$. This gives the desired contradiction, since $x^{i}(h, \cdot)$ gives the same utility as $\mu^{i}$ for all $h$.

To conclude the proof, we show that $\hat{\mu}^{i}$ can be purchased at prices $\hat{p}$. Suppose for some $i$ this is not the case, that $\hat{\mu}^{i}$ costs more than the transfer to that type. Then since $\hat{\mu}$ is socially feasible, for some other type $i$ ' expenditure on $\hat{\mu}^{i}$ must be less than the transfer payment. But by Lemma 5.1, such a type could use the extra income to purchase a better lottery than $\hat{\mu}^{i}$, which we have shown cannot happen.

\section{The Stand-in Consumer Economy}

We now prove the welfare theorems and the existence of an equilibrium. From the equivalence of the sunspot and lottery equilibria, it is sufficient to do so in either of the two types of economies. Each approach, however, poses its own complications. The sunspot economy has a net trade set that is complicated and non-convex. The lottery economy has a net trade set that is convex but infinite dimensional. One approach is that of Prescott and Townsend [20], which is to work directly with theorems for infinite dimensional economies. The alternative pursued here leads to finite dimensional and mathematically simpler proofs by observing that the household problem of maximizing utility subject to a budget constraint can be broken in two parts. The first part, since the 
cost of a set of lotteries is simply the cost of the expected net trades it uses, is to think of the household as purchasing an expected net trade vector. The second part is to think of the household as choosing the set of lotteries that maximizes utility subject to this expected net trades constraint. This utility depends only on the expected net trade vector, which is finite dimensional, so in effect reduces the economy to a finite one.

Specifically, we consider net trade vectors $y^{i} \in \mathfrak{R}^{J}$. The set of interest are net trade vectors that are consistent with feasible trading plans of type $i$ households:

$$
\left.Y^{i} \equiv \text { Closure(ConvexHull }\left\{y^{i} \in \mathfrak{R}^{J} \mid \exists x_{s}^{i} \in X_{s}^{i}, y^{i}=\sum_{s \in S^{i}} \pi_{s}^{i} x_{s}^{i}\right\}\right) .
$$

Given that a bundle $y^{i} \in Y^{i}$ has been purchased, how much utility can a type $i$ household get? The answer is given by

$$
U^{i}\left(y^{i}\right)=\sup \int \sum_{s \in S^{i}} \pi_{s}^{i} u_{s}^{i}\left(x_{s}^{i}\right) d \mu_{s}^{i}\left(x_{s}^{i}\right)
$$

subject to support $\mu_{s}^{i} \subseteq X_{s}^{i}, \lambda^{i} \int \sum_{s \in S^{i}} \pi_{s}^{i} x_{s}^{i} d \mu_{s}^{i}\left(x_{s}^{i}\right) \leq y^{i}, u_{s}^{i}\left(\mu_{s}^{i}\right)-u_{s}^{i}\left(\mu_{s^{i}}^{i}\right) \geq 0, s^{\prime} \in F^{i}(s)$.

This construct will be most useful if we can replace the sup with a max, so that there is at least one lottery that actually yields the utility $U^{i}\left(y^{i}\right)$.

Lemma 7.1 If the boundary condition holds, then

$U^{i}\left(y^{i}\right)=\max \int \sum_{s \in S^{i}} \pi_{s}^{i} u_{s}^{i}\left(x_{s}^{i}\right) d \mu_{s}^{i}\left(x_{s}^{i}\right)$ subject to support $\mu_{s}^{i} \subseteq X_{s}^{i}, \int \sum_{s \in S^{i}} \pi_{s}^{i} x_{s}^{i} d \mu_{s}^{i}\left(x_{s}^{i}\right) \leq y^{i}$, $u_{s}^{i}\left(\mu_{s}^{i}\right)-u_{s}^{i}\left(\mu_{s^{\prime}}^{i}\right) \geq 0, s^{\prime} \in F^{i}(s)$.

Proof: By Lemma 5.1 we can assume that there is a sequence of sets of lotteries with each lottery having support at $k^{i}+J+2$ points converging to the sup. Let $x_{s}^{i \ell}, \mu_{s}^{i \ell}$ be the points and probabilities in this sequence. This has a convergent subsequence on the extended real line. Because $X_{s}^{i}$ is bounded below, any component of $x_{s}^{i \ell}$ that converges to $\infty$ has corresponding probability converging to zero. By the boundary condition the limit of expected utility for such a point is also zero. So the limit set of lotteries places weight only on finitely many points, and gives the same utility and satisfies the feasibility and incentive conditions. It is the optimal set of lotteries.

We now study trade in the economy, by considering $I$ consumers with utility functions $U^{i}$ and consumption sets $Y^{i}$. We refer to consumer $i$ as the stand-in consumer, 
as he represents all households of type $i$. The stand-in consumer makes purchases on behalf of the ex ante identical households he represents, then allocates the purchases to individual households by means of an optimal lottery. Notice the role played here by the assumption that all households of a given type are ex ante identical: there is no ambiguity about how a lottery should be chosen to allocate resources among individual households.

In the stand-in consumer economy, an allocation $y$ is a vector $y^{i} \in Y^{i}$ for each type. The allocation is socially feasible if $\sum_{i} y^{i} \leq 0$. A stand-in consumer equilibrium with transfers consists of a non-zero price vector $\hat{p} \in \mathfrak{R}_{+}^{J}$, and a socially feasible allocation $\hat{y}$. For each type $i, y^{i}$ should maximize $U^{i}\left(y^{i}\right)$ subject to $\hat{p} \cdot y^{i} \leq \hat{p} \cdot \hat{y}^{i}$, $y^{i} \in Y^{i}$. An endowment equilibrium and Pareto efficiency are defined in the obvious way. Notice that equilibria in the stand-in consumer economy are equivalent to equilibria in the lottery economy in a direct and simple way. Given a lottery equilibrium $\hat{\mu}, \hat{p}$, we can compute the expected resources used by the equilibrium lottery $y^{i}=\lambda^{i} \int \sum_{s \in S^{i}} \pi_{s}^{i} x_{s}^{i} d \mu_{s}^{i}\left(x_{s}^{i}\right)$. Clearly $\hat{y}, \hat{p}$ are a stand-in consumer equilibrium. Conversely, given a stand-in consumer equilibrium $\hat{y}, \hat{p}$, we can use Lemma 7.1 to find for each stand-in consumer an optimal set of lotteries $\hat{\mu}^{i}$, and it is clear that $\hat{\mu}, \hat{p}$ are a lottery equilibrium.

To prove the welfare and existence theorems for the sunspot economy and lottery economy, it suffices to prove them for the stand-in consumer economy. As this is a finite dimensional pure exchange economy, this follows from verifying standard properties of utility functions and consumption sets.

Lemma 7.2 Utility $U^{i}$ is continuous, concave, and, if non-satiation holds, strictly increasing. The net trade set has $0 \in Y^{i}$ and is closed, convex and bounded below. If the cheaper point assumption holds, then there is a point $0>y^{i} \in Y^{i}$.

\section{Exclusivity and Incentive Constraints}

We have already pointed out that incentive constraints demand exclusivity of contracts: although households of a particular type are ex ante identical, ex post they realize different values of the idiosyncratic shocks, and would want to trade with one another. The use of sunspots or lotteries introduces another dimension in which households are ex post different: even households who realize the same idiosyncratic 
shock will have different ex post net trades, as some win and some lose in the lottery. This raises the question of whether even households with the same idiosyncratic state will want to trade in equilibrium. The answer is that, in the absence of incentive constraints, for example, when there are indivisibilities, households do not want to trade. Consequently, it is only in economies with incentive constraints that we require exclusivity.

For simplicity, we limit attention to lotteries that have countable support. This will be the case if the consumption sets are discrete, as they may be with indivisibilities. From the proof of Lemma 7.2, we also know that for any lottery equilibrium, there is another lottery equilibrium yielding exactly the same utility and with each type consuming the same aggregate resources, in which the support of the lottery is finite. The result we prove holds more generally, but the proof of the most general case is more technical.

Lemma 8.1: Assume that for all $s \in S^{i}, F_{s}^{i}=\{s\}$, so that there are no incentive constraints. Suppose that p supports the upper contour set of $U^{i}$ at $y^{i}$, support $\mu_{s}^{i} \subseteq X_{s}^{i}$, $\int \sum_{s \in S^{i}} \pi_{s}^{i} x_{s}^{i} d \mu_{s}^{i}\left(x_{s}^{i}\right) \leq y^{i}$ and, $\int \sum_{s \in S^{i}} u_{s}^{i}\left(x_{s}^{i}\right) d \mu_{s}^{i}\left(x_{s}^{i}\right)=U^{i}\left(y^{i}\right)$, and that support $\mu_{s}^{i}$ is countable. Let $x_{s}^{i} \in X_{s}^{i}$ be such that $\mu_{s}^{i}\left(x_{s}^{i}\right)>0$. Then $p$ supports the upper contour set of $u_{s}^{i}$ at $x_{s}^{i}$.

Proof: Suppose conversely to the Lemma that there is $\tilde{x}_{s}^{i} \in X_{s}^{i}$ with $u_{s}^{i}\left(\tilde{x}^{i}\right)>u^{i}\left(x_{s}^{i}\right)$, and $p \cdot\left(\tilde{x}_{s}^{i}-x_{s}^{i}\right) \leq 0$. Consider $\tilde{\mu}_{s}^{i}$ defined by

$$
\tilde{\mu}_{s}^{i}\left(z_{s}^{i}\right)=\left\{\begin{array}{cc}
\mu_{s}^{i}\left(z_{s}^{i}\right) & z_{s}^{i} \neq x_{s}^{i}, \tilde{x}_{s}^{i} \\
0 & z_{s}^{i}=x_{s}^{i} \\
\mu_{s}^{i}\left(\tilde{x}_{s}^{i}\right)+\mu_{s}^{i}\left(x_{s}^{i}\right) & z_{s}^{i}=\tilde{x}_{s}^{i}
\end{array}\right.
$$

For $s^{\prime} \neq s$ take $\tilde{\mu}_{s}^{i}=\mu_{s^{i}}^{i}$. Set $w^{i}=\int \sum_{s \in S^{i}} \pi_{s}^{i} \widehat{x}_{s}^{i} d \tilde{\mu}_{s}^{i}\left(\hat{x}_{s}^{i}\right)$, where $\hat{x}_{s}^{i}$ is the dummy variable of integration. Then $p \cdot\left(w^{i}-y^{i}\right) \leq 0$ and $u^{i}\left(w^{i}\right)>u^{i}\left(y^{i}\right)$, a contradiction.

\section{Risk Aversion and Lotteries}

While in principle lotteries may be useful when there are incentive constraints, in many practical examples, equilibrium lotteries are degenerate. This is not a necessary conclusion: Cole [7] gives a robust example in which lotteries are used to sort high 
marginal utility from low marginal utility states. Cole's example has the odd feature, however, that the high marginal utility households, who we would generally think of as having low endowments, are less risk averse than low marginal utility households. In this section, we show that in the more plausible case of decreasing absolute risk aversion, equilibrium lotteries are in fact degenerate. This is the case in our initial example.

We now specialize to the case of an economy in which there are no indivisibilities. We assume that each household of type $i$ in state $s$ has an endowment of $\omega_{s}^{i} \in \mathfrak{R}_{++}^{J}$, and a utility function for consumption $v_{s}^{i}\left(c_{s}^{i}\right)$ that is strictly increasing and concave. The net trade set $X_{s}^{i}=\left\{x_{s}^{i} \mid x_{s}^{i} \geq-\omega_{s}^{i}\right\}$ allows no indivisibilities, and the utility function $u_{s}^{i}\left(x_{s}^{i}\right)=v_{s}^{i}\left(\omega_{s}^{i}+x_{s}^{i}\right)$. In this setting, given a lottery $\mu_{s}^{i}$ over net trades, we define the certainty equivalent $\bar{c}_{s}^{i}\left(\mu_{s}^{i}\right) \in \mathfrak{R}_{++}^{J}$ to be the fraction of the expected consumption from the lottery that is equivalent to the expected utility from the lottery, $\bar{c}_{s}^{i}\left(\mu_{s}^{i}\right)=\theta_{s}^{i}\left(\omega_{s}^{i}+\int x_{s}^{i} d \mu_{s}^{i}\left(x_{s}^{i}\right)\right)$, where

$$
v_{s}^{i}\left(\theta_{s}^{i}\left(\omega_{s}^{i}+\int x_{s}^{i} d \mu_{s}^{i}\left(x_{s}^{i}\right)\right)\right)=\int v_{s}^{i}\left(\omega_{s}^{i}+x_{s}^{i}\right) d \mu_{s}^{i}\left(x_{s}^{i}\right)
$$

and, since utility is assumed concave, $0<\theta_{s}^{i} \leq 1$.

State Independent Preferences: $v_{s}^{i}\left(c_{s}^{i}\right)=v^{i}\left(c_{s}^{i}\right)$

Decreasing Absolute Risk Aversion: If $z_{s}^{i}>0$, then

$$
v^{i}\left(z_{s}^{i}+\bar{c}_{s}^{i}\left(\mu_{s}^{i}\right)\right)<\int v^{i}\left(z_{s}^{i}+\omega_{s}^{i}+x_{s}^{i}\right) d \mu_{s}^{i}\left(x_{s}^{i}\right) .
$$

This says that the certainty equivalent is an increasing function of consumption, or equivalently, that the risk premium is declining. It is straightforward to check that in the case of a single good, this is equivalent to the usual definition.

We will show that, if (for all types) preferences are state independent and exhibit non-increasing absolute risk aversion, then there is always an equilibrium with degenerate lotteries. It is convenient to prove this using a weaker condition that does not require state independent utilities.

Generalized Decreasing Risk Aversion: If $s^{\prime} \in F_{s}^{i}$ then

$$
v_{s}^{i}\left(\omega_{s}^{i}+\bar{c}_{s}^{i}\left(\mu_{s}^{i}\right)-\omega_{s}^{i}\right)<\int v_{s}^{i}\left(\omega_{s}^{i}+x_{s}^{i}\right) d \mu_{s^{\prime}}^{i}\left(x_{s}^{i}\right)
$$


From the assumption of feasible misrepresentation this definition makes sense, since $X_{s}^{i} \supseteq X_{s}^{i}$, means that $\mu_{s}^{i}$, is in fact a lottery in $X_{s}^{i}$; this assumption combined with state independent decreasing absolute risk aversion also implies generalized decreasing risk aversion. Basically, this assumption says that a household that actually has state $s '$ is more risk averse than a household that is masquerading as state $s$.

Theorem 9.1 With generalized decreasing absolute risk aversion (and no indivisibilities), every solution to the stand-in consumer problem is a point mass on a single point for each s.

Proof: Let $\hat{\mu}_{s}^{i}$ be lotteries that solve the stand-in consumer problem. Consider the alternative degenerate lottery in that puts mass one on $\bar{c}_{s}^{i}\left(\hat{\mu}_{s}^{i}\right)-\omega_{s}^{i}$. This lottery consumes no more resources than $\hat{\mu}_{s}^{i}$ since $v_{s}^{i}$ is concave, and, if households tell the truth, they yield exactly the same utility. Moreover, the generalized decreasing risk aversion condition means that any incentive constraint satisfied under $\hat{\mu}_{s}^{i}$ is satisfied as well under the new plan. Finally, if any of the $\hat{\mu}_{s}^{i}$ are non-degenerate, then, since the risk premium is assumed strictly positive, strictly fewer resources are consumed by the degenerate alternative, which contradicts Lemma 5.1.

\section{An Example}

Having shown that decreasing absolute risk aversion leads to degenerate lotteries, we turn now to an example in which increasing absolute risk aversion leads to nondegenerate examples. As in Cole [7], we focus on the case where there are two states, a single good and a single type. For notational convenience, we omit the superscript $i$. With two states, which we denote $g, b$, and a single good, we denote the endowments as $\omega_{g}>\omega_{b}$. In addition, we assume voluntary public endowments. This means that households can optionally reveal their endowments. Consequently, the sets of feasible reports are $F_{g}=\{g, b\}, F_{b}=\{b\}$.

Let $\left\{x_{s \xi}\right\}$ be a finite set of points on which $\mu_{s}$ puts weight. Stand-in consumer utility is then given by

$$
\max _{\mu} \sum_{s \in S} \sum_{\xi} \pi_{s} v\left(\omega_{s}+x_{s \xi}\right) \mu_{s}\left(x_{s \xi}\right)
$$




$$
\begin{gathered}
\text { subject to } \\
\sum_{s \in S} \sum_{\xi} \pi_{s} x_{s \xi} \mu_{s}\left(x_{s \xi}^{i}\right) \leq 0 \\
\sum_{\xi} v\left(\omega_{g}+x_{g \xi}\right) \mu_{g}\left(x_{g \xi}\right)-\sum_{\xi} v\left(\omega_{g}+x_{b \xi}\right) \mu_{g}\left(x_{b \xi}\right) \geq 0 \\
\sum_{\xi} \mu_{s}\left(x_{s \xi}\right)=1 \\
\mu_{s}\left(x_{s \xi}\right) \geq 0 .
\end{gathered}
$$

This is a linear program that can be solved on any grid $\left\{x_{s \xi}\right\}$. Lemma 5.1(c) says that there will be a solution that places weight on at most 4 different points for each $\mu_{s}$. As the grid is refined, the set of approximate solutions will approach the set of exact solutions to the problem; if the original grid is carefully chosen, it is be possible to find an exact solution on the grid. Notice that this is the general sort of linear programming problem that we need to solve to find the optimal lottery allocation for a household type in the stand-in consumer economy. In the general case, there are $J$ resource constraints, which replace the 0 on the right-hand side with $y_{j}^{i}$, and $k^{i}$ incentive constraints.

To have non-degenerate lotteries requires increasing absolute risk aversion. A convenient family with this property is that of quadratic utility functions. Consider the quadratic utility function $v(c)=78 c-c^{2}$ with endowments $\omega_{g}=30, \omega_{b}=10$ and probabilities $\pi_{g}=\pi_{b}=1 / 2$. It can be verified that a solution to the linear programming problem defining the stand-in consumer utility is a degenerate lottery in the good state with $\mu_{g}(-1)=1$, and in the bad state the non-degenerate lottery $\mu_{b}(7)=1 / 2, \mu_{b}(-5)=1 / 2$. The mean transfer in the good state is -1 , with the mean transfer in the bad state of +1 . The transfer in the bad state involves a large gamble between +7 and -5 , however, and the well-endowed household prefers to avoid this risk. There are multiple solutions to this example. For example, $\mu_{g}(-1)=1$, $\mu_{b}(1)=7 / 16, \mu_{b}(9)=9 / 32, \mu_{b}(-7)=9 / 32$ is also a solution.

To verify that all solutions to our example involve non-degenerate lotteries, we provide a sufficient condition under which a non-degenerate lottery improves welfare, so that the solution to the stand-in consumer problem will necessarily be non-degenerate. If there is only one good, then the only degenerate lottery that satisfies the incentive constraint and does not lower welfare is autarky: $\mu_{g}(0)=1, \mu_{b}(0)=1$. We now search for conditions under which a small lottery can improve welfare while satisfying the incentive 
constraint. Suppose that we replace $\mu_{b}(0)=1$ with a small lottery $x$ with mean $\bar{x}$ and variance $\sigma_{x}^{2}$. To be specific, let $\mu_{b}\left(x^{\prime}\right)$ be a lottery with mean 0 and variance 1 (for example, $\mu_{b}(1)=1 / 2$ and $\left.\mu_{b}(-1)=1 / 2\right)$, and let $x=\bar{x}+\sigma_{x} x$. We set $\mu_{g}\left(x_{g}\right)=1$, where, to maintain social feasibility, we require that

$$
x_{g}=-\frac{\pi_{b}}{\pi_{g}} \bar{x} .
$$

The incentive constraint holding exactly can be written

$$
v\left(\omega_{g}-\frac{\pi_{b}}{\pi_{g}} \bar{x}\right)=E v\left(\omega_{g}+x\right) .
$$

To second order, this constraint can be written approximately as

$$
\begin{aligned}
& v\left(\omega_{g}\right)-v^{\prime}\left(\omega_{g}\right) \frac{\pi_{b}}{\pi_{g}} \bar{x}+\frac{1}{2} v^{\prime \prime}\left(\omega_{g}\right)^{2}\left(\frac{\pi_{b}}{\pi_{g}}\right)^{2} \bar{x}^{2} \\
& =v\left(\omega_{g}\right)+v^{\prime}\left(\omega_{g}\right) \bar{x}+\frac{1}{2} v^{\prime \prime}\left(\omega_{g}\right)\left(\sigma_{x}^{2}+\bar{x}^{2}\right) .
\end{aligned}
$$

Using this equation we can now solve, at least to second order, for the variance of the lottery for which the incentive constraint is satisfied as a function of the mean of the lottery:

$$
\sigma_{x}^{2}(\bar{x})=-\frac{2 v^{\prime}\left(\omega_{g}\right)}{\pi_{g} v^{\prime \prime}\left(\omega_{g}\right)} \bar{x}-\frac{\pi_{g}^{2}-\pi_{b}^{2}}{\pi_{g}^{2}} \bar{x}^{2} .
$$

A second order Taylor series expansion allows us to approximate the ex ante utility of a small lottery that satisfies the incentive constraint as a function of its mean:

$$
\begin{gathered}
V(\bar{x})=\pi_{g}\left[v\left(\omega_{g}\right)-v^{\prime}\left(\omega_{g}\right) \frac{\pi_{b}}{\pi_{g}} \bar{x}+\frac{1}{2} v^{\prime \prime}\left(\omega_{g}\right)\left(\frac{\pi_{b}}{\pi_{g}}\right)^{2} \bar{x}^{2}\right] \\
+\pi_{b}\left[v\left(\omega_{b}\right)+v^{\prime}\left(\omega_{b}\right) \bar{x}+\frac{1}{2} v^{\prime \prime}\left(\omega_{b}\right)\left(\sigma_{x}^{2}(\bar{x})+\bar{x}^{2}\right)\right] .
\end{gathered}
$$

Differentiating with respect to $\bar{x}$, we find

$$
V^{\prime}(0)=\pi_{b}\left(v^{\prime}\left(\omega_{b}\right)-v^{\prime}\left(\omega_{g}\right)\right)-\frac{\pi_{b}}{\pi_{g}} \frac{v^{\prime}\left(\omega_{g}\right) v^{\prime \prime}\left(\omega_{b}\right)}{v^{\prime \prime}\left(\omega_{g}\right)} .
$$

If this expression is strictly positive, as it is in our numerical example, then introducing a small lottery with a positive mean and a variance just large enough to make the incentive 
constraint hold increases welfare. Consequently, the degenerate lottery cannot be the solution to the stand-in consumer problem, and a non-degenerate lottery must be used in equilibrium.

It is worth pointing out two features of this example and our calculations. First, notice that, if utility exhibits decreasing absolute risk aversion, then

$$
-\frac{v^{\prime \prime}\left(\omega_{b}\right)}{v^{\prime}\left(\omega_{b}\right)}>-\frac{v^{\prime \prime}\left(\omega_{g}\right)}{v^{\prime}\left(\omega_{g}\right)}
$$

and it is impossible for $V^{\prime}(0)$ to be positive at a small non-degenerate lottery. Even if there is increasing absolute risk aversion, however, there may not be non-degenerate lotteries: for this, increasing absolute risk aversion is necessary but not sufficient. Second, notice that, if utility is quadratic, then the formula we obtain for $V(\bar{x})$ is exact, and not just a good approximation for small lotteries. In fact, it has been by maximizing this function that we have obtained the numerical example.

The intuition for the presence of multiple equilibria in our numerical example is simple: The function $V(\bar{x})$ is maximized by a lottery in the bad state with mean 1 and variance 36. This pins down expected utility in both states, but there is an infinite number of lotteries in the bad state with this mean and variance, which is all that matter for a quadratic utility function. The trick is to make the consumer in the good state indifferent between reporting the good state and making a transfer of 1 and reporting the bad state and receiving the transfer.

We can use our numerical example to illustrate some issues related to sunspot allocations. One sunspot allocation, equivalent to the lottery allocation $\mu_{g}(-1)=1$ and $\mu_{b}(7)=1 / 2, \mu_{b}(-5)=1 / 2$ is $x_{g}(h, \sigma)=-1$ and

$$
x_{b}(h, \sigma)=\left\{\begin{aligned}
7 & \text { if }(\sigma+h) \bmod 1 \geq 1 / 2 \\
-5 & \text { if }(\sigma+h) \bmod 1<1 / 2 .
\end{aligned}\right.
$$

This allocation is far from unique, however, since any way of relating the sunspot variable $\sigma$ to the index of the household $h$ that results in each household receiving +7 with probability $1 / 2$ and -5 with probability $1 / 2$ works just as well. Yet another optimal sunspot allocation is 


$$
x_{b}(h, \sigma)=\left\{\begin{aligned}
7 & \text { if }(\sigma+2 h) \bmod 1 \leq 1 / 2 \text { and } h \leq 1 / 2 \\
-5 & \text { if }(\sigma+2 h) \bmod 1>1 / 2 \text { and } h \leq 1 / 2 \\
1 & \text { if }(\sigma+2 h-1) \bmod 1 \leq 7 / 16 \text { and } h>1 / 2 \\
9 & \text { if } 7 / 16<(\sigma+2 h-1) \bmod 1 \leq 23 / 32 \text { and } h>1 / 2 \\
-7 & \text { if } 23 / 32<(\sigma+2 h-1) \bmod 1 \text { and } h>1 / 2 .
\end{aligned}\right.
$$

This is an example of the sort discussed in Section 6 in which the sunspot allocation induces different lotteries for different households. In this example, the mean lottery is $\tilde{\mu}_{b}(7)=1 / 4, \quad \tilde{\mu}_{b}(-5)=1 / 4, \quad \tilde{\mu}_{b}(1)=7 / 32, \quad \tilde{\mu}_{b}(9)=9 / 64, \quad \tilde{\mu}_{b}(-7)=9 / 64$, which is also a solution to the stand-in consumer problem.

\section{Ex Ante Lotteries}

We have studied a model in which sunspots occur after households announce their state. From Cole [7] we know that the model in which sunspots occur before households announce their states is quite different. The more general case allows for both types of sunspots. See Fellingham, Kwon, and Newman [10] for a discussion. Specifically, let $\left(\sigma_{0}, \sigma_{1}\right) \in[0,1]^{2}$ be uniform independent sunspots occurring before and after the announcement of the idiosyncratic state. We know that there is a 1-1 continuous (and therefore measurable) map from $m:[0,1] \rightarrow[0,1]^{2}$, the Peano curve is an example of such a map. So we can define $\sigma=m^{-1}\left(\sigma_{0}, \sigma_{1}\right)$. In this way, we can map prices and allocations in the two-sunspot model to prices and allocation in the ex post sunspot model; we assume that the probability distribution over sunspots is that induced by $m^{-1}$. Our final result shows that if the price/allocation pair form an equilibrium in the two-sunspot model, the corresponding price/allocation pair is an equilibrium in the ex post sunspot model

Theorem 11.1: If $\hat{x}\left(\sigma_{0}, \sigma_{1}\right), \hat{q}\left(\sigma_{0}, \sigma_{1}\right)$ are an equilibrium in the two-sunspot model, then $\hat{x}(m(\sigma)), \hat{q}(m(\sigma))$ is an equilibrium in the ex post sunspot model.

Proof: This is really just a matter of checking that the incentive constraints remain satisfied in the ex post model - the ex post joint distribution of net trades and prices is the same as in the two-sunspot model by construction, so utilities and budget constraints are the same. Since, however, the incentive constraint must hold for each $\sigma_{0}$ in the twosunspot model, and must hold only when averaged over $\sigma_{0}$ in the ex post sunspot model, 
the incentive constraints in the ex post model follow from those in the two-sunspot model. 


\section{Appendix}

Lemma 5.1 (c) For any incentive compatible set of lotteries $\mu^{i}$ there is another incentive compatible set of lotteries $\tilde{\mu}^{i}$ using the same aggregate resources, yielding the same utility, and each lottery $\tilde{\mu}_{s}^{i}$ having support on $k^{i}+J+2$ points.

Proof: Define a map

$$
z:\left(\times_{s \in S^{i}} X_{s}^{i}\right) \rightarrow \mathfrak{R}^{k^{i}+J+1}
$$

taking the first $k^{i}$ components equal to the benefit to telling the truth $u_{s}^{i}\left(x_{s}^{i}\right)-u_{s}^{i}\left(x_{s}^{i}\right)$ for $s^{\prime} \in F_{s}^{i}, s^{\prime} \neq s$; the next $J$ components equal to the aggregate resources used

$$
\lambda^{i} \sum_{s \in S^{i}} \pi_{s}^{i} x_{s}^{i},
$$

the $k^{i}+J+1$ component equal to utility

$$
\sum_{s \in S^{i}} \pi_{s}^{i} u_{s}^{i}\left(x_{s}^{i}\right)
$$

Define the set

$$
Z=z\left(\times_{s \in S^{i}} X_{s}^{i}\right)
$$

and consider probability measures $\eta$ on $Z$. Let $\hat{\eta}$ be the measure derived by treating the set of lotteries $\left\{\mu_{s}^{i}\right\}$ as independent to induce a probability distribution on $\times_{s \in S^{i}} X_{s}^{i}$, then mapping to $Z$ by $z$. Consider the linear equation in $\eta \int z d \eta(z)=\int z d \hat{\eta}(z)$. This implies that $\int z d \hat{\eta}(z)$ is in the convex hull of $Z$. Consequently, by Caratheodory's Theorem for $\ell=1, \ldots, k^{i}+J+2$ there are points $z^{\ell} \in Z$ and non-negative weights $\eta^{\ell}$ such that

$$
\sum_{\ell} z^{\ell} \eta^{\ell}=\int z d \hat{\eta}(z)
$$

For each $\ell$ choose

$$
\left(x_{s}^{i \ell}\right)_{s \in S^{i}} \in z^{-1}\left(z^{\ell}\right) \text {. }
$$

Then $\tilde{\mu}_{s}^{i}\left(x_{s}^{i \ell}\right)$ equal the sum of probabilities $\eta^{\ell}$ such that $x_{s}^{i \ell}$ is chosen is the desired measure. 


\section{References}

1. F. Alvarez. and U. J. Jermann. Efficiency, equilibrium, and asset pricing with risk of default, Econometrica, 68 (2000), 775-798..

2. R. Arnott and J. E. Stiglitz. Randomization with asymmetric information, RAND J. Econ. 19 (1987), 344-362.

3. A. Bennardo. "Competitive Equilibria and Second Best Allocations of Multicommodity Economies with Moral Hazard: The Case of Perfect Verifiability of Trades," DELTA, Paris, 1997.

4. A. Bennardo and P. A. Chiappori. "Competition, Positive Profits and Market Clearing under Asymmetric Information,” University of Chicago, 1997.

5. A. Bisin and D. Guaitoli. "Inefficiency of Competitive Equilibrium with Asymmetric Information and Financial Intermediaries,” New York University, 1995.

6. R. T. Boylan. Laws of large numbers for dynamical systems with randomly matched individuals, J. Econ. Theory 57 (1992), 473-504.

7. H. L. Cole. Comment: General competitive analysis in an economy with private information," Int. Econ. Rev. 30 (1989), 249-252.

8. H. L. Cole and N. R. Kocherlakota. "Efficient Allocations with Hidden Income and Storage," Federal Reserve Bank of Minneapolis Research Department Staff Report 238, 1997

9. H. L. Cole and E. C. Prescott. Valuation equilibria with clubs. J. Econ. Theory $\mathbf{7 4}$ (1997), 19-39.

10. J. C. Fellingham, Y. K. Kwon, and D. P. Newman. Ex ante randomization in agency models, RAND J. Econ. 15 (1984), 290-301.

11. R. J. Garratt. Decentralizing lottery allocations in markets with indivisible commodities, Econ. Theory 5 (1995), 295-314.

12. R. J. Garratt, D. T. Keister, C. Qin, and K. Shell. On sunspot equilibrium prices, J. Econ. Theory, forthcoming.

13. J. D. Geanakoplos. An introduction to general equilibrium with incomplete asset markets, J. Math. Econ. 19 (1990), 1-38.

14. G. D. Hansen. Indivisible labor and the business cycle, J. Monet. Econ. 16 (1985), 309-327. 
15. T. J. Kehoe and D. K. Levine. Debt constrained asset markets, Rev. Econ. Stud. 60 (1993), 865-888.

16. T. J. Kehoe and D. K. Levine. Liquidity constrained markets versus debt constrained markets, Econometrica, forthcoming.

17. N. R. Kocherlakota. Implications of efficient risk sharing without commitment, Rev. Econ. Stud. 63 (1996), 595-609.

18. D. Kreuger. Risk sharing in economies with incomplete markets. Stanford University, 2000.

19. E. C. Prescott and R. M. Townsend. Pareto optima and competitive equilibria with adverse selection and moral hazard, Econometrica 52 (1984), 21-45.

20. E. C. Prescott and R. M. Townsend. General competitive analysis in an economy with private information, Int. Econ. Rev. 25 (1984), 1-20.

21. E. S. Prescott and R. M. Townsend. Firms as clubs in Arrow-Debreu markets with private information. University of Chicago, 1999.

22. R. Rogerson. Indivisible labor, lotteries, and equilibrium, J. Monet. Econ. 21 (1988), $3-16$.

23. K. Shell and R. Wright. Indivisibilities, lotteries and sunspot equilibria, Econ. Theory 3 (1993), 1-17.

24. H. Uhlig. A law of large numbers for large economies, Econ. Theory 8 (1996), 41-50. 